\title{
2-氯喹啉-3-甲醛缩氨基胍衍生物的合成及其抗菌活性评价
}

\author{
高智敏 ${ }^{\dagger}$ 王田田 ${ }^{\dagger}$ 李深圳 万慧琪 \\ 王刚吴银涁 邓先清宋明霞* \\ (井冈山大学基础医学与药学学院 吉安 343009)
}

\begin{abstract}
摘要 为寻找新的高活性的广谱抗菌剂, 通过活性拼接原理, 设计合成了 8 个未见文献报道的 2-氯喹啉-3-甲醛缩氨基 胍衍生物, 并通过光谱方法确证结构. 所合成化合物对所选的菌种(除了 Salmonella typhimurium 2421 和 Pseudomonas aeruginosa 2742)均显示出了较好的抑制活性，最低抑菌浓度值(MIC)范围大都为 $2.0 \sim 16 \mu \mathrm{g} / \mathrm{mL}$. 化合物 $4 \mathbf{h}$ 显示出最 好的广谱抗菌活性，其对抗所选的 6 种菌株的最低抑菌浓度值均为 $2.0 \mu \mathrm{g} / \mathrm{mL}$. 其对抗 Staphylococcus aureus KCTC 503 和两种耐药菌(Methicillin-resistant Staphylococcus aureus CCARM 3167 和 Quinolone-resistant Staphylococcus aureus CCARM 3505)的活性要优于或相当于阳性对照药加替沙星、莫西沙星、诺氟沙星和苯唑西林.
\end{abstract}

关键词 缩氨基胍; 2-氯喹啉; 抗菌活性; 耐药菌

\section{Synthesis and Antibacterial Activity Evaluation of (2-Chloroquinolin- 3-yl)methyleneamino Guanidine Derivatives}

\author{
Gao, Zhimin ${ }^{\dagger} \quad$ Wang, Tiantian ${ }^{\dagger} \quad$ Li, Shenzhen Wan, Huiqi Wang, Gang \\ Wu, Yinbin Deng, Xianqing Song, Mingxia* \\ (Basic Medical and Pharmacy College, Jinggangshan University, Ji'an 343009)
}

\begin{abstract}
In order to find new antibacterial agent with wide spectra and high activities, 8 new (2-chloroquinolin-3-yl)methyleneamino guanidine derivatives were synthesized based on combination principles, whose structures were then identified by spectral methods. All of the compounds provided exhibited good inhibitory activities against the strains chosen, except Salmonella typhimurium 2421 and Pseudomonas aeruginosa 2742, of which MICs were mostly in the range of $2.0 \sim 16$ $\mu \mathrm{g} / \mathrm{mL}$. The compound $\mathbf{4 h}$ showed the best broad-spectrum antibacterial activities, whose MIC value was $2.0 \mu \mathrm{g} / \mathrm{mL}$ against six strains. The inhibitory activities of $\mathbf{4 h}$, against Staphylococcus aureus KCTC 503 and two drug-resistance bacterias (Methicillin-resistant Staphylococcus aureus CCARM 3167 and Quinolone-resistant Staphylococcus aureus CCARM 3505), were superior or equal to positive controls gatifloxacin, moxifloxacin, norfloxacin and oxacillin.

Keywords alkylene aminoguanidine; 2-chloroquinoline; antibacterial activity; drug-resistance bacteria
\end{abstract}

21 世纪以来, 细菌耐药性问题在全球范围内都出 现, 并且日益严重 ${ }^{[1]}$. 根据英国首相经济学顾问的调查 报告, 如果细菌耐药不加遏制, 到 2050 年全球每年将有 1000 万人死于耐药菌感染，每年经济损失将高达 100 万 亿美元 ${ }^{[2]}$. 在中国, 细菌耐药问题同样严重. 在 2009 年 的中国部分医院调查中发现: 超过 $60 \%$ 患者的金黄色葡 萄球菌菌株为甲氧西林耐药(Methicillin-resistant Staphylococcus aureus, MRSA); 超过 $60 \%$ 的铜绿假单胞菌菌
株多药耐药，包括头狍他啶、环丙沙星、阿米卡星和亚 胺培南; 有 $70 \%$ 的大肠杆菌耐氟喹诺酮类药物, 为世界 之最 ${ }^{[3,4]}$. 遗憾的是，新型抗生素的研发并没有伴随着耐 药性细菌的增加而增加. 近 50 年来, 只有两个新型抗生 素(利奈唑胺与达托霉素)被批准用于治疗革兰氏阳性菌 感染，而用于治疗革兰氏阴性菌(G-菌)感染的新药一个 也没有 ${ }^{[5]}$. 多药耐药的感染加上缺乏新的抗菌药物，导 致临床医生重新考虑使用多黏菌素作为最后的治疗手

*E-mail: freexiaoxiao83@aliyun.com

Received April 19, 2016; revised May 31, 2016; published online June 8, 2016.

Project supported by the National Natural Science Foundation of China (No. 21562028), and the Doctoral Foundation of Jinggangshan University (No. JZB1317).

国家自然科学基金(No. 81560561)和井冈山大学博士启动基金(No. JZB1317)资助项目.

†共同第一作者(These authors contributed equally to this work). 
段, 而这些药物由于存在神经毒性和肾毒性, 已经多年 未在临床使用 ${ }^{[6,7]}$. 因此, 寻找具有新结构和新靶点的新 型抗菌药物尤其是抗耐药菌和抗 G-药物已迫在眉睫.

喹啉又称苯并吡啶, 是一个重要的荎状含氮杂环 物. 许多喹啉化合物都是重要医药中间体, 而且许多含 喹啉环的药物被不断开发出来, 如抗疮药物氯喹、解热 镇痛药物辛可芬、局部麻醉药物盐酸地布卡因; 抗阿米 巴病药喹碘仿、抗菌素药物克菌定等. 喹啉类衍生物的 其他生物活性也相继被报道, 如抗菌 ${ }^{[8 \sim 10]}$ 、抗疼 ${ }^{[11]}$ 、抗 癌 ${ }^{[12]}$ 、抗 $\mathrm{HIV}^{[13]}$ 、抗炎等 ${ }^{[14]}$. 氨基胍(Aminoguanidine, $\mathrm{AG}$ )又称为胍基肼, 1892 年由 Thiele 首次合成. 氨基胍 由于富含电子对, 是一个优良的金属配位基, 因此在金 属配合物抗癌抗病毒药物研究中表现突出 ${ }^{[15,16]}$. 近年 来, 有研究报道氨基胍衍生物具有抗菌活性, 其抗菌活 性与氨基胍的配位作用和氢键结合能力相关 ${ }^{[17]}$. 为了 寻找新结构和新靶点的抗菌活性化合物, 本文将氨基艮 和喹啉片段进行拼合, 设计合成了一系列喹啉缩氨基胍 化合物, 期望可以得到具有广谱抗菌活性的新化学实 体. 对这些新化合物进行了多种菌株包括革兰氏阴性 菌、革兰氏阳性菌、耐药菌和真菌的体外抑菌活性评价.

\section{1 结果与讨论}

以 6-羟基-喹啉酮为起始原料, 与氯芐进行亲核取 代反应, 然后在 $\mathrm{POCl}_{3}$ 与 $\mathrm{DMF}$ 作用下发生威尔斯迈克哈克反应, 得到 2-氯-6-芐氧基-喹啉-3-醛( $\mathbf{3 a} \sim 3 \mathbf{h}$ ). 最 后, 3a $\sim 3 \mathrm{~h}$ 与氨基胍碳酸氢盐缩合得到一系列最终产物 6-取代苄氧基-2-氯喹啉-3-甲醛缩氨基胍盐酸盐 $4 \mathbf{a} \sim \mathbf{4 h}$, 如 Scheme 1 所示. 所有化合物结构经过核磁共振和质 谱等谱学方法得到确证.
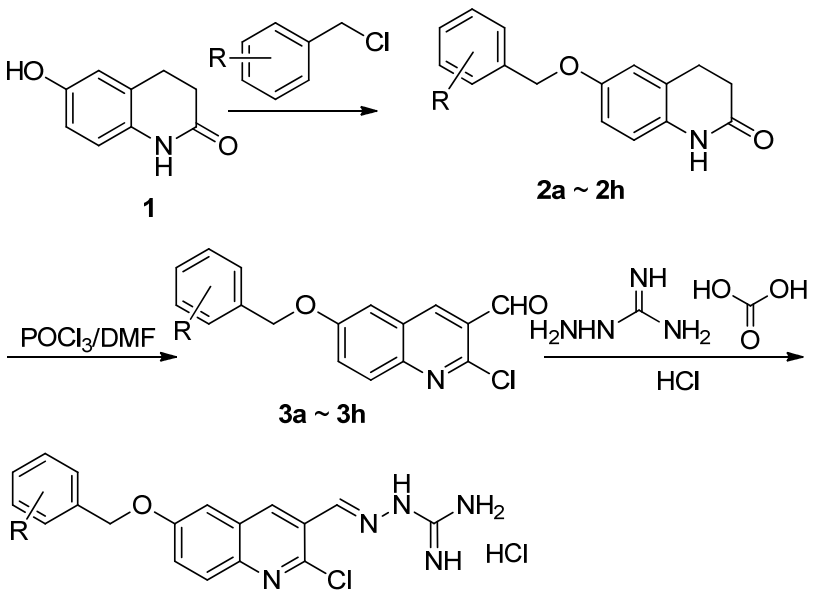

4a $\sim \mathbf{4 h}$

a: $R=H, b: R=2-F, c: R=3-F, d: R=4-F$

e: $\mathrm{R}=2-\mathrm{Cl}, \mathbf{f}: \mathrm{R}=3-\mathrm{Cl}, \mathbf{g}: \mathrm{R}=4-\mathrm{Cl}, \mathbf{h}: \mathrm{R}=4-\mathrm{CH}_{3}$

图式 12 -氯喹啉-3-甲醛缩氨基胍衍生物的合成路线

Scheme 1 Synthetic route of (2-chloroquinolin-3-yl)-methyleneamino guanidine derivatives

\section{1 波谱解析}

产物的波谱特征以化合物 $4 \mathrm{a}$ 为例，在 ${ }^{1} \mathrm{H} N \mathrm{NMR}$ 中， 芐基中亚甲基两个氢受到苯基和氧原子的影响, 其吸收 峰出现在较高化学位移 $\delta 5.26$ 处, 但与苯环的吸收峰可 以较好地区分; 芳香氢区域在 $\delta 7.35 \sim 7.91$ 范围内出现 多个吸收峰, 是苄基苯环上和喹啉苯环上 8 个氢的吸收, 这两个苯环的吸收峰相互重叠不能很好地区分解析; $\delta$ 7.99 处有一个钝峰, 是胍基氮原子上四个氢的吸收峰, 胍基在这里之所以是一个单峰是因为其结构发生了互 变使共轭链延长, 末端形成了两个相同的 $\mathrm{NH}_{2}$; 吡啶环 上 4 位的氢受吡啶环和双键的影响出现在化学位移 9.16 处. ${ }^{13} \mathrm{C}$ NMR 谱也准确地给出了该化合物结构的各种信 息, 恰好是 16 种不同化学环境的碳. 盐酸盐在质谱测定 中不稳定, 通常获得的是脱去盐酸之后的离子峰. 在本 实验中, 化合物 $4 \mathrm{a}$ 离去氯离子之后的理论分子量为 354.1116, 高分辨质谱仪实际测定值为 354.1112, 结合 氢谱可以确证其分子结构.

\section{2 抑菌活性}

本试验采用系列稀释法测定目标化合物对所选菌 株的抑制活性，抑菌活性用最低抑菌浓度 MIC 值进行 表示, 测试结果见表 1.

化合物 $4 \mathrm{a} \sim 4 \mathrm{~h}$ 对所选的 5 种革兰氏阳性菌均显示 出一定程度的抑制活性. 其中化合物 $4 \mathrm{~b}$ 和 $4 \mathrm{~h}$ 对金黄色 葡萄球菌(Staphylococcus aureus RN 4220)活性最好, 抗 菌活性是诺氟沙星的 2 倍, 与苯唑西林抗菌活性相当. 对金黄色葡萄球菌(Staphylococcus aureus KCTC 503), 化合物抗菌活性稍弱一些, MIC 值大都在 $8.0 \sim 32$ $\mu \mathrm{g} / \mathrm{mL}$ 范围内，只有化合物 $4 \mathrm{~h}$ 的活性达到了 $2.0 \mu \mathrm{g} / \mathrm{mL}$, 其抗菌活性是加替沙星的 2 倍, 与诺氟沙星和苯唑西林 抗菌活性相当. 对变形链球菌 (Streptococcus mutans 3289), 化合物 4a 活性最好, 但要弱于阳性对照药加替 沙星和莫西沙星. 对所选的两种耐药金黄色葡萄球菌 (Methicillin-resistant Staphylococcus aureus CCARM 3167 和 Quinolone-resistant Staphylococcus aureus CCARM 3505), 化合物 $\mathbf{4 a} \sim \mathbf{4 h}$ 均表现出较好的活性, 其中对耐甲氧西林金黄色葡萄球菌 (MRSA CCARM 3167)活性最好, 化合物 $\mathbf{4 b} 、 \mathbf{4 c} 、 \mathbf{4 e} 、 \mathbf{4 f}$ 和 $\mathbf{4 h}$ 的 MIC 值均为 $2.0 \mu \mathrm{g} / \mathrm{mL}$, 是苯唑西林的 32 倍, 诺氟沙星的 4 倍, 与加替沙星活性相当, 但稍弱于莫西沙星. 对抗耐 喹诺酮金黄色葡萄球菌( $R R S A$ CCARM 3505), 只有化 合物 $4 \mathbf{h}$ 的 MIC 值达到了 $2.0 \mu \mathrm{g} / \mathrm{mL}$, 优于阳性对照药加 替沙星 $(8.0 \mu \mathrm{g} / \mathrm{mL})$ 、莫西沙星 $(4.0 \mu \mathrm{g} / \mathrm{mL})$ 和诺氟沙星 $(>$ $64 \mu \mathrm{g} / \mathrm{mL})$, 稍弱于苯唑西林 $(1.0 \mu \mathrm{g} / \mathrm{mL})$. 其他化合物的 最低抑菌浓度大都为 $8.0 \mu \mathrm{g} / \mathrm{mL}$. 
表 1 化合物 $\mathbf{4 a} \sim \mathbf{4 h}$ 对所选临床菌株的抑制活性 $(\mathrm{MIC}, \mu \mathrm{g} / \mathrm{mL})^{a}$

Table 1 Inhibitory activity (MIC,$\mu \mathrm{g} / \mathrm{mL})$ of compounds $\mathbf{4 a} \sim \mathbf{4 h}$ against clinical isolates selected

\begin{tabular}{|c|c|c|c|c|c|c|c|c|c|c|}
\hline \multirow{2}{*}{ Compd. } & \multirow{2}{*}{$\mathrm{R}$} & \multicolumn{5}{|c|}{ Gram-positive strains } & \multicolumn{3}{|c|}{ Gram-negative strains } & \multirow{2}{*}{$\frac{\text { Fungj }}{7535^{j}}$} \\
\hline & & $4220^{b}$ & $503^{c}$ & $3167^{d}$ & $3505^{e}$ & $3289^{f}$ & $1924^{g}$ & $2421^{h}$ & $2742^{i}$ & \\
\hline $4 a$ & $\mathrm{H}$ & 4.0 & 8.0 & 4.0 & 8.0 & 2.0 & 4.0 & $>64$ & $>64$ & 4.0 \\
\hline $4 b$ & $2-\mathrm{F}$ & 2.0 & 8.0 & 2.0 & 4.0 & 4.0 & 4.0 & $>64$ & $>64$ & 4.0 \\
\hline $4 c$ & $3-\mathrm{F}$ & 4.0 & 8.0 & 2.0 & 8.0 & 8.0 & 4.0 & $>64$ & $>64$ & 4.0 \\
\hline $4 d$ & $4-\mathrm{F}$ & 16 & 32 & 4.0 & 32 & 16 & 16 & $>64$ & $>64$ & 16 \\
\hline $4 e$ & $2-\mathrm{Cl}$ & 4.0 & 8.0 & 2.0 & 4.0 & 4.0 & 4.0 & $>64$ & $>64$ & 8.0 \\
\hline $4 f$ & $3-\mathrm{Cl}$ & 4.0 & 8.0 & 2.0 & 8.0 & 16 & 4.0 & $>64$ & $>64$ & 4.0 \\
\hline $4 \mathrm{~g}$ & $4-\mathrm{Cl}$ & 8.0 & 16 & 4.0 & 16 & 64 & 8.0 & $>64$ & $>64$ & 8.0 \\
\hline $4 h$ & $4-\mathrm{CH}_{3}$ & 2.0 & 2.0 & 2.0 & 2.0 & 4.0 & 2.0 & $>64$ & $>64$ & 2.0 \\
\hline Gatifloxacin & & 0.25 & 4.0 & 2.0 & 8.0 & 0.50 & 2.0 & 0.50 & 1.0 & N.D* \\
\hline Moxifloxacin & & 0.25 & 2.0 & 1.0 & 4.0 & 0.25 & 2.0 & 0.50 & 1.0 & N.D* \\
\hline Norfloxacin & & 2.0 & 2.0 & 8.0 & $>64$ & N.D* & N.D* & N.D* & N.D* & N.D* \\
\hline Oxacillin & & 1.0 & 1.0 & $>64$ & 1.0 & N.D* & N.D* & N.D* & N.D* & N.D* \\
\hline Fluconazole & & N.D* & N.D* & N.D* & N.D* & N.D* & N.D* & N.D* & N.D* & 1.0 \\
\hline Itraconazole & & N.D* & N.D* & N.D* & N.D* & N.D* & N.D* & N.D* & N.D* & 0.060 \\
\hline
\end{tabular}

${ }^{a}$ 所有实验平行进行 3 次, MIC 值取其平均值, N.D 为未检测. ${ }^{b}$ Staphylococcus aureus RN 4220, ${ }^{c}$ Staphylococcus aureus KCTC 503, ${ }^{d}$ Methicillin-resistant Staphylococcus aureus CCARM 3167, ${ }^{e}$ Quinolone-resistant Staphylococcus aureus CCARM 3505, ${ }^{f}$ Streptococcus mutans $3289,{ }^{g}$ Escherichia coli KCTC 1924, ${ }^{h}$ Salmonella typhimurium 2421, ${ }^{i}$ Pseudomonas aeruginosa $2742,{ }^{j}$ Candida albicans 7535.

对所选的 3 种革兰氏阴性菌, 目标化合物对鼠伤寒 沙门氏菌 (Salmonella typhimurium 2421) 和绿脓杆菌 (Pseudomonas aeruginosa 2742)在 $64 \mu \mathrm{g} / \mathrm{mL}$ 下无抑制活 性, 但其对大肠杆菌(Escherichia coli KCTC 1924)的活 性较好, MIC 值大都为 $4.0 \mu \mathrm{g} / \mathrm{mL}$. 其中化合物 $\mathbf{4 h}$ 的活 性最好, MIC 值为 $2.0 \mu \mathrm{g} / \mathrm{mL}$, 活性与加替沙星和莫西沙 星相当.

化合物 $4 \mathrm{a} \sim \mathbf{4 h}$ 对所选的真菌白色念球菌 (Candida albicans 7535)亦表现出一定的活性, MIC 值大都为 4.0 或 $8.0 \mu \mathrm{g} / \mathrm{mL}$, 其中化合物 $\mathbf{4 h}$ 的活性最好, MIC 值为 2.0 $\mu \mathrm{g} / \mathrm{mL}$, 但其活性仍弱于阳性对照药氟康唑和依曲康唑.

分析其构效关系, 对于氟取代和氯取代的化合物, 对抗革兰氏阳性菌和阴性菌的活性顺序大致为 2-氯 (氟) $\geqslant 3$-氯(氟) $>4$-氯(氟); 对于真菌 Candida albicans 7535 , 氯取代化合物活性顺序稍有不同, 活性顺序为 3氯>2-氯, 4-氯. 这表明取代基的位置对活性的影响呈 现出一定的规律性. 2 -氯(氟)和 3 -氯(氟)取代的化合物 整体抗菌活性要优于无取代的化合物(除了 S. mutans 3289). 4-甲基取代的化合物抗菌活性最好, 其对抗所选 的 6 种菌株的 MIC 值均为 $2.0 \mu \mathrm{g} / \mathrm{mL}$, 要优于氯或氟取 代化合物. 对于 S. mutans 3289, 无取代化合物活性最 好, MIC 值为 $2.0 \mu \mathrm{g} / \mathrm{mL}$, 推测可能苯环上引入基团不利 于化合物与细菌内靶标的结合而导致活性降低.

\section{2 结论}

本文设计合成了 8 个未见文献报道的新化合物, 并 对其进行了体外抗菌活性评价. 药理结果显示, 除了鼠 伤寒沙门氏菌(S. typhimurium 2421) 和绿脓杆菌(P. aeru- ginosa 2742), 8 个化合物对所选的革兰氏阴性菌、革兰 氏阳性菌、耐药菌和真菌都显示出明显的抑制作用, 部 分化合物的抑菌 MIC 值达到 $2.0 \mu \mathrm{g} / \mathrm{mL}$. 该系列化合物 的抗菌活性的发现为新的广谱抗菌剂的研发提供了新 的结构骨架.

\section{3 实验部分}

\section{1 仪器与试剂}

红外光谱用 FTIR1730 型光谱仪测定, 采用澳化钾 压片法(PerkineElmer, Waltham, MA, USA); 核磁共振用 BURKER AV-300 型核磁共振仪测定，四甲基硅(TMS) 为内标, 溶剂用 $\mathrm{CDCl}_{3}$ 或 DMSO- $d_{6}$ (Bruker, Switzerland); 高分辨质谱采用 MALDI-TOF/TOF 型质谱仪 (Bruker Daltonik, Germany); 熔点采用毛细管法测定(温 度计未经校正).

\subsection{6 -取代苄氧基-2-氯喹啉-3-甲醛缩氨基胍盐酸盐} (4a $4 \mathrm{~h}$ )的合成

6-取代茮氧基-3,4-二氢喹啉-2(1H)-酩(2a $\sim 2 \mathbf{h})$ 和 6取代芐氧基-2-氯喹啉-3-甲醛(3a $\sim 3 \mathbf{h})$ 按照文献[18]方法 进行制备. 将 6-着基-3,4-二氢喹啉-2 $(1 H)$-酮 $(4.9 \mathrm{mmol}$ )、 相应的氯芐 $(5.9 \mathrm{mmol})$ 、无水碳酸钾 $(9.8 \mathrm{mmol})$ 加入含有 $20 \mathrm{~mL}$ 乙腈的 $50 \mathrm{~mL}$ 圆底烧瓶中, $90{ }^{\circ} \mathrm{C}$ 下回流反应 $4.0 \sim 14 \mathrm{~h}$. 通过 TLC 检测, 反应结束后将反应液倒入 $120 \mathrm{~mL}$ 水中，搅拌, 静置析出沉淀, 过滤, 并用水洗涤 3 次, 干燥得到白色固体 $\mathbf{2 a} \sim \mathbf{2 h}$. 无需纯化用于下一步 实验.

取 $N, N$-二甲基甲酰胺 $(30 \mathrm{mmol})$ 于圆底烧瓶中, 冰 浴下搅拌, 逐滴加入 $\mathrm{POCl}_{3}(4.0 \mathrm{mmol})$, 搅拌 $5.0 \mathrm{~min}$ 后 
缓慢加入化合物 $\mathbf{2 a} \sim \mathbf{2 h}(1.0 \mathrm{mmol})$, 室温反应 $30 \mathrm{~min}$ 后 升温至 $110{ }^{\circ} \mathrm{C}$, 反应 $2.0 \sim 4.0 \mathrm{~h}$. 通过 TLC 检测, 反应结 束后将反应液倒入 $100 \mathrm{~mL}$ 冰水中, 搅拌, 析出沉淀, 过 滤, 冰水洗涤 3 次, 干燥得到黄色固体化合物 $\mathbf{3 a} \sim \mathbf{3 h}$, 该固体无需纯化用于下一步实验.

取化合物 $3 \mathbf{a} \sim \mathbf{3 h}(1.0 \mathrm{mmol})$ 和氨基胍碳酸氢盐(1.2 $\mathrm{mmol}$ )于圆底烧瓶中, 加入 $10 \mathrm{~mL}$ 无水乙醇溶解, 滴加 10 滴浓 $\mathrm{HCl}, 70{ }^{\circ} \mathrm{C}$ 下搅拌反应 $4.0 \mathrm{~h}$. 减压旋干溶剂, 柱 层析分离 [洗脱剂: $V\left(\mathrm{CH}_{2} \mathrm{Cl}_{2}\right): V\left(\mathrm{CH}_{3} \mathrm{OH}\right)=30: 1.0 \sim$ $10 ： 1.0$ 得到黄色粉末状固体 $\mathbf{4 a} \sim \mathbf{4 h}$.

6-(苄氧基)-2-氯喹啉-3-甲醛缩氨基胍盐酸盐(4a): 产率 48\%. m.p. 205 207 ${ }^{\circ} \mathrm{C} ;{ }^{1} \mathrm{H}$ NMR $\left(\mathrm{CDCl}_{3}, 300\right.$ $\mathrm{MHz}) \delta: 5.26\left(\mathrm{~s}, 2 \mathrm{H}, \mathrm{CH}_{2}\right), 7.35 \sim 7.91(\mathrm{~m}, 8 \mathrm{H}, \mathrm{Ar}-\mathrm{H})$, 7.99 (br s, 4H, guanidyl-H), $8.57(\mathrm{~s}, 1 \mathrm{H}, \mathrm{CH}=\mathrm{C}), 9.16(\mathrm{~s}$, $1 \mathrm{H}, \mathrm{CH}=\mathrm{N}), 12.45(\mathrm{~s}, 1 \mathrm{H}, \mathrm{HCl}) ;{ }^{13} \mathrm{C} \mathrm{NMR}\left(\mathrm{CDCl}_{3}, 75\right.$ MHz) $\delta: 157.6,156.1,146.3,143.8,142.0,136.8,135.8$, $129.9,129.0,128.5,128.4,128.3,126.0,125.2,107.6$, 70.2; IR (KBr) v: 3308, 3169, 1682, $1620 \mathrm{~cm}^{-1}$; ESI-HRMS calcd for $\mathrm{C}_{18} \mathrm{H}_{17} \mathrm{Cl}_{2} \mathrm{~N}_{5} \mathrm{O}\left([\mathrm{M}-\mathrm{Cl}]^{+}\right) 354.1116$, found 354.1112 .

6-(2-氟芳氧基)-2-氯喹啉-3-甲醛缩氨基胍盐酸盐 (4b): 产率 49\%. m.p. 214 215 ${ }^{\circ} \mathrm{C} ;{ }^{1} \mathrm{H}$ NMR $\left(\mathrm{CDCl}_{3}\right.$, $300 \mathrm{MHz}) \delta: 5.26\left(\mathrm{~s}, 2 \mathrm{H}, \mathrm{CH}_{2}\right), 7.39 \sim 7.90(\mathrm{~m}, 7 \mathrm{H}, \mathrm{Ar}-\mathrm{H})$, 8.05 (br s, 4H, guanidyl-H), $8.57(\mathrm{~s}, 1 \mathrm{H}, \mathrm{CH}=\mathrm{C}), 9.16(\mathrm{~s}$, $1 \mathrm{H}, \mathrm{CH}=\mathrm{N}), 12.54(\mathrm{~s}, 1 \mathrm{H}, \mathrm{HCl}) ;{ }^{13} \mathrm{C} \mathrm{NMR}\left(\mathrm{CDCl}_{3}, 75\right.$ $\mathrm{MHz}) \delta: 162.1\left(\mathrm{~d},{ }^{1} J_{\mathrm{c}-\mathrm{f}}=241 \mathrm{~Hz}\right), 157.4,155.9,146.3$, $143.9,142.1,136.2,135.9\left(\mathrm{~d},{ }^{3} J_{\mathrm{c}-\mathrm{f}}=6.8 \mathrm{~Hz}\right), 133.1,130.1$ $\left(\mathrm{d},{ }^{3} J_{\mathrm{c}-\mathrm{f}}=7.4 \mathrm{~Hz}\right), 129.9,129.0\left(\mathrm{~d},{ }^{2} J_{\mathrm{c}-\mathrm{f}}=21.5 \mathrm{~Hz}\right), 128.4$, $125.9,125.1,115.6$ (d, $\left.{ }^{2} J_{\mathrm{c}-\mathrm{f}}=20.8 \mathrm{~Hz}\right), 107.7,69.3$; IR (KBr) v: 3300, 3127, 1686, $1622 \mathrm{~cm}^{-1}$; ESI-HRMS calcd for $\mathrm{C}_{18} \mathrm{H}_{16} \mathrm{FCl}_{2} \mathrm{~N}_{5} \mathrm{O}\left([\mathrm{M}-\mathrm{Cl}]^{+}\right) 372.1022$, found 372.1011 .

6-(3-氟芳氧基)-2-氯喹啉-3-甲醛缩氨基胍盐酸盐 (4c): 产率 52\%. m.p. 213 $214{ }^{\circ} \mathrm{C} ;{ }^{1} \mathrm{H}$ NMR $\left(\mathrm{CDCl}_{3}, 300\right.$ $\mathrm{MHz}) \delta: 5.30\left(\mathrm{~s}, 2 \mathrm{H}, \mathrm{CH}_{2}\right), 7.17 \sim 7.92(\mathrm{~m}, 7 \mathrm{H}, \mathrm{ArH}), 7.96$ (br s, 4H, guanidyl-H), $8.57(\mathrm{~s}, 1 \mathrm{H}, \mathrm{CH}=\mathrm{C}), 9.16(\mathrm{~s}, 1 \mathrm{H}$, $\mathrm{CH}=\mathrm{N}), 12.44(\mathrm{~s}, 1 \mathrm{H}, \mathrm{HCl}) ;{ }^{13} \mathrm{C} \mathrm{NMR}\left(\mathrm{CDCl}_{3}, 75 \mathrm{MHz}\right)$ $\delta: 162.7\left(\mathrm{~d},{ }^{1} J_{\mathrm{c}-\mathrm{f}}=247 \mathrm{~Hz}\right), 157.4,156.1,146.4,143.8$, $141.9,139.7\left(\mathrm{~d},{ }^{3} J_{\mathrm{c}-\mathrm{f}}=7.6 \mathrm{~Hz}\right), 135.8,131.1\left(\mathrm{~d},{ }^{3} J_{\mathrm{c}-\mathrm{f}}=8.1\right.$ $\mathrm{Hz}), 129.9,128.4,126.1,125.1,124.1\left(\mathrm{~d},{ }^{4} J_{\mathrm{c}-\mathrm{f}}=2.6 \mathrm{~Hz}\right)$, $115.3\left(\mathrm{~d},{ }^{2} J_{\mathrm{c}-\mathrm{f}}=20.8 \mathrm{~Hz}\right), 114.8\left(\mathrm{~d},{ }^{2} J_{\mathrm{c}-\mathrm{f}}=21.7 \mathrm{~Hz}\right), 107.7$, 69.3; IR (KBr) $v$ : 3302, 3134, 1684, $1620 \mathrm{~cm}^{-1}$. ESI-HRMS calcd for $\mathrm{C}_{18} \mathrm{H}_{16} \mathrm{FCl}_{2} \mathrm{~N}_{5} \mathrm{O}\left([\mathrm{M}-\mathrm{Cl}]^{+}\right)$ 372.1022, found 372. 1022.

6-(4-氟芳氧基)-2-氯喹啉-3-甲醛缩氨基胍盐酸盐 (4d): 产率 55\%. m.p. 225 226 ${ }^{\circ} \mathrm{C} ;{ }^{1} \mathrm{H}$ NMR $\left(\mathrm{CDCl}_{3}\right.$,
$300 \mathrm{MHz}) \delta: 5.24\left(\mathrm{~s}, 2 \mathrm{H}, \mathrm{CH}_{2}\right), 7.22 \sim 7.91(\mathrm{~m}, 7 \mathrm{H}, \mathrm{ArH})$, 8.01 (br s, 4H, guanidyl-H), $8.58(\mathrm{~s}, 1 \mathrm{H}, \mathrm{CH}=\mathrm{C}), 9.17$ (s, $1 \mathrm{H}, \mathrm{CH}=\mathrm{N}), 12.47(\mathrm{~s}, 1 \mathrm{H}, \mathrm{HCl}) ;{ }^{13} \mathrm{C} \mathrm{NMR}\left(\mathrm{CDCl}_{3}, 75\right.$ $\mathrm{MHz}) \delta: 162.4\left(\mathrm{~d},{ }^{1} J_{\mathrm{c}-\mathrm{f}}=245 \mathrm{~Hz}\right), 157.5,156.0,146.3$, $143.8,142.0,135.9,133.0\left(\mathrm{~d},{ }^{4} J_{\mathrm{c}-\mathrm{f}}=3.0 \mathrm{~Hz}\right), 130.6(\mathrm{~d}$, $\left.{ }^{3} J_{\mathrm{c}-\mathrm{f}}=8.3 \mathrm{~Hz}\right), 129.9,128.4,126.0,125.2,115.8\left(\mathrm{~d},{ }^{2} J_{\mathrm{c}-\mathrm{f}}=\right.$ $21.3 \mathrm{~Hz}), 107.7,69.5$; IR (KBr) v: 3306, 3130, 1680, 1620 $\mathrm{cm}^{-1}$. ESI-HRMS calcd for $\mathrm{C}_{18} \mathrm{H}_{16} \mathrm{FCl}_{2} \mathrm{~N}_{5} \mathrm{O}\left([\mathrm{M}-\mathrm{Cl}]^{+}\right)$ 372.1022, found 372. 1022 .

6-(2-氯苄氧基)-2-氯喹啉-3-甲醛缩氨基胍盐酸盐 (4e): 产率 41\%. m.p. 221 222 ${ }^{\circ} \mathrm{C} ;{ }^{1} \mathrm{H} \mathrm{NMR}\left(\mathrm{CDCl}_{3}, 300\right.$ $\mathrm{MHz}) \delta: 5.30\left(\mathrm{~s}, 2 \mathrm{H}, \mathrm{CH}_{2}\right), 7.42 \sim 7.91(\mathrm{~m}, 7 \mathrm{H}, \mathrm{Ar}-\mathrm{H})$, 8.06 (br s, 4H, guanidyl-H), 8.57 (s, $1 \mathrm{H}, \mathrm{CH}=\mathrm{C}), 9.19$ (s, $1 \mathrm{H}, \mathrm{CH}=\mathrm{N}), 12.56(\mathrm{~s}, 1 \mathrm{H}, \mathrm{HCl}) ;{ }^{13} \mathrm{C} \mathrm{NMR}\left(\mathrm{CDCl}_{3}, 75\right.$ $\mathrm{MHz}) \delta$ : 157.3, 155.9, 146.4, 143.9, 142.0, 136.0, 134.1, $132.9,130.4,130.3,130.0,129.9,128.4,127.9,126.0$, 125.1, 107.5, 67.7; IR (KBr) v: 3345, 3148, 1682, 1618 $\mathrm{cm}^{-1}$; ESI-HRMS calcd for $\mathrm{C}_{18} \mathrm{H}_{16} \mathrm{Cl}_{3} \mathrm{~N}_{5} \mathrm{O}\left([\mathrm{M}-\mathrm{Cl}]^{+}\right)$: 388.0726, found 388.0726.

6-(3-氯苄氧基)-2-氯喹啉-3-甲醛缩氨基胍盐酸盐 (4f): 产率 40\%. m.p. $270 \sim 271{ }^{\circ} \mathrm{C} ;{ }^{1} \mathrm{H} \mathrm{NMR}\left(\mathrm{CDCl}_{3}, 300\right.$ $\mathrm{MHz}) \delta: 5.28\left(\mathrm{~s}, 2 \mathrm{H}, \mathrm{CH}_{2}\right), 7.39 \sim 7.92(\mathrm{~m}, 7 \mathrm{H}, \mathrm{ArH}), 8.06$ (br.s, 4H, guanidyl-H), $8.57(\mathrm{~s}, 1 \mathrm{H}, \mathrm{CH}=\mathrm{C}), 9.17(\mathrm{~s}, 1 \mathrm{H}$, $\mathrm{CH}=\mathrm{N}), 12.57(\mathrm{~s}, 1 \mathrm{H}, \mathrm{HCl}) ;{ }^{13} \mathrm{C} \mathrm{NMR}\left(\mathrm{CDCl}_{3}, 75 \mathrm{MHz}\right)$ $\delta: 157.4,155.9,146.4,143.9,142.1,139.4,135.9,133.7$, $130.9,129.9,128.5,128.4,127.8,126.7,126.0,125.1$, 107.7, 69.3; IR (KBr) v: 3362, 3184, 1678, $1624 \mathrm{~cm}^{-1}$; ESI-HRMS calcd for $\mathrm{C}_{18} \mathrm{H}_{16} \mathrm{Cl}_{3} \mathrm{~N}_{5} \mathrm{O}\left([\mathrm{M}-\mathrm{Cl}]^{+}\right)$: 388.0726, found 388.0723.

6-(4-氯苄氧基)-2-氯喹啉-3-甲醛缩氨基胍盐酸盐 (4g): 产率 53\%. m.p. 252 253 ${ }^{\circ} \mathrm{C} ;{ }^{1} \mathrm{H} \mathrm{NMR}\left(\mathrm{CDCl}_{3}, 300\right.$ $\mathrm{MHz}) \delta: 5.31\left(\mathrm{~s}, 2 \mathrm{H}, \mathrm{CH}_{2}\right), 7.27 \sim 7.92(\mathrm{~m}, 7 \mathrm{H}, \mathrm{ArH}), 8.00$ (br s, 4H, guanidyl-H), $8.59(\mathrm{~s}, 1 \mathrm{H}, \mathrm{CH}=\mathrm{C}), 9.19(\mathrm{~s}, 1 \mathrm{H}$, $\mathrm{CH}=\mathrm{N}), 12.55(\mathrm{~s}, 1 \mathrm{H}, \mathrm{HCl}) ;{ }^{13} \mathrm{C} \mathrm{NMR}\left(\mathrm{CDCl}_{3}, 75 \mathrm{MHz}\right)$ $\delta: 157.4,156.0,146.4,143.9,142.0,135.9,131.0,130.0$, 128.4 126.0, 125.1, 123.7, 116.0, 115.8, 107.6, 64.5; IR (KBr) v: 3308, 3148, 1680, $1620 \mathrm{~cm}^{-1}$; ESI-HRMS calcd for $\mathrm{C}_{18} \mathrm{H}_{16} \mathrm{Cl}_{3} \mathrm{~N}_{5} \mathrm{O}\left([\mathrm{M}-\mathrm{Cl}]^{+}\right)$388.0726, found 388.0728.

6-(4-甲基苠氧基)-2-氯喹啉-3-甲醛缩氨基胍盐酸盐 (4h): 产率 57\%. m.p. 213 214 ${ }^{\circ} \mathrm{C} ;{ }^{1} \mathrm{H} \mathrm{NMR}\left(\mathrm{CDCl}_{3}\right.$, $300 \mathrm{MHz}) \delta: 5.20\left(\mathrm{~s}, 2 \mathrm{H}, \mathrm{CH}_{2}\right), 7.19 \sim 7.88(\mathrm{~m}, 7 \mathrm{H}, \mathrm{ArH})$, 8.06 (br s, 4H, guanidyl-H), $8.57(\mathrm{~s}, 1 \mathrm{H}, \mathrm{CH}=\mathrm{C}), 9.16(\mathrm{~s}$, $1 \mathrm{H}, \mathrm{CH}=\mathrm{N}), 12.59(\mathrm{~s}, 1 \mathrm{H}, \mathrm{HCl}) ;{ }^{13} \mathrm{C} \mathrm{NMR}\left(\mathrm{CDCl}_{3}, 75\right.$ MHz) $\delta: 157.6,155.9,147.2,146.2,143.8,142.1,137.8$, $135.9,133.7,129.8,129.5,128.3,125.9,125.2,107.6$, 
70.1, 21.2; IR (KBr) v: 3302, 3190, 1678, $1622 \mathrm{~cm}^{-1}$; ESI-HRMS calcd for $\mathrm{C}_{19} \mathrm{H}_{19} \mathrm{Cl}_{2} \mathrm{~N}_{5} \mathrm{O}\left([\mathrm{M}-\mathrm{Cl}]^{+}\right) 368.1273$, found 368.1274 .

\section{3 抗菌活性实验}

本试验所测菌株包括: 金黄色葡萄球菌 (Staphylococcus aureus RN 4220 和 Staphylococcus aureus KCTC 503)、耐药金黄色葡萄球菌( Quinolone-resistant Staphylococcus aureus CCARM 3505 和 Methicillinresistant Staphylococcus aureus CCARM 3167)、变形链球 菌(Streptococcus mutans 3289)、大肠杆菌(Escherichia coli KCTC 1924)、鼠伤寒沙门氏菌 (Salmonella typhimurium 2421)、绿脓杆菌(Pseudomonas aeruginosa 2742)和白色念球菌(Candida albicans 7535). 采用连续 稀释法测定化合物对所选细菌生长的抑制作用, 获得化 合物对抗不同菌株的最低抑菌浓度. 最低抑菌浓度 (Minimal inhibitory concentration, MIC) 是抑制细菌生长 所需药物的最低浓度. 本实验选取加替沙星、莫西沙星、 苯唑西林、诺氟沙星、氟康唑和依曲康唑作为阳性对照 药.

测试用细菌先于 Mueller-Hinton Broth (MHB)培养 基培养至对数期, 将培养好的细菌用培养基稀释成 $10^{5} \mathrm{CFU} / \mathrm{mL}$ 浓度接种于 96 孔细胞培养板中 (最终体积为 $200 \mu \mathrm{L}$ ). 样品和对照药溶解于二甲基亚砜(DMSO)中, DMSO 的最终浓度不得高于 $0.05 \%$. 采用连续稀释法获 得一系列样品浓度为 $64 \sim 1 \mu \mathrm{g} / \mathrm{mL}, 37{ }^{\circ} \mathrm{C}$ 下培养 $24 \mathrm{~h}$. 用酶标仪在 $650 \mathrm{~nm}$ 测培养前后吸光度, 根据吸光度的 变化计算得到最低抑菌浓度即 MIC 值. 所有实验平行 进行 3 次. 化合物 $\mathbf{4 a} \sim \mathbf{4 h}$ 与阳性对照药的体外抗菌活性 见表 1 .

辅助材料(Supporting Information) 化合物的核磁共 振图谱、高分辨质谱和红外光谱. 这些材料可以免费从 本刊(http://sioc-journal.cn/)上下载.

\section{References}

[1] Livermore, D. M. J. Antimicrob. Chemother. 2009, 64(Supp1. 1), i29.

[2] Azeredo da Silveira, S.; Perez, A. Expert Rev. Anti-Infect. Ther. 2015, 13, 531.

[3] Hvistendahl, M. Science 2012, 336, 795.

[4] Yezli, S.; Li, H. Int. J. Antimicrob. Agents 2012, 40, 389.

[5] Xu, Z. Q.; Xu, Z. Y. Acta Pharmaceutica Sinica 2013, 48, 993 (in Chinese). (徐泽奇, 徐泽宇, 药学学报, 2013, 48, 993.)

[6] Kofteridis, D. P.; Alexopoulou, C.; Valachis, A.; Maraki, S.; Dimopoulou, D.; Georgopoulos, D.; Samonis, G. Clin. Infect. Dis. 2010, $51,1238$.

[7] Velkov, T.; Thompson, P. E.; Nation, R. L.; Li, J. J. Med. Chem. 2010, 53, 1898.

[8] Thomas K. D.; Adhikari, A. V.; Telkar, S.; Chowdhury, I. H.; Mahmood, R.; Pal, N. K.; Row, G.; Sumesh, E. Eur. J. Med. Chem. 2011, 46, 5283.

[9] Eswaran, S.; Adhikari, A. V.; Shetty, N. S. Eur. J. Med. Chem. 2009, 44, 4637.

[10] Kategaonkar, A. H.; Shinde, P. V.; Kategaonkar, A. H.; Pasale, S. K.; Shingate, B. B.; Shangare, M. S. Eur. J. Med. Chem. 2010, 45, 3142 .

[11] Lilienkampf, A.; Mao, J.; Wan, B.; Wang, Y.; Franzblau, S. G.; Kozikowski, A. P. J. Med. Chem. 2009, 52, 2109.

[12] Denny, W. A.; Wilson, W. R.; Ware, D. C.; Atwell, G. J.; Milbank, J. B.; Stevenson, R. J. WO 2002059122, 2002 [Chem. Abstr. 2002, 137, 149339].

[13] Strekowski, L.; Mokrosz, J. L.; Honkan, V. A.; Czarny, A.; Cegla, M. T.; Patterson, S. E.; Wydra, R. L.; Schinazi, R. F. J. Med. Chem. 1991, 34, 1739.

[14] Nasveld, P.; Kitchener, S. Trans. R. Soc. Trop. Med. Hyg. 2005, 99, 2.

[15] Ferreira, E. G.; Wilke, D. V.; Jimenez, P. C.; de Oliveira, J. R.; Pessoa O. D.; Silveira, E. R.; Viana, F. A.; Pessoa, C.; de Moraes, M. O.; Hajdu, E.; Costa-Lotufo L. V. Chem. Biodiversity 2011, 8, 1433.

[16] Ren, S.; Wang, R.; Komatsu, K.; Bonaz-Krause, P.; Zyrianov, Y.; McKenna C. E.; Csipke C.; Tokes Z. A.; Lien E. J. J. Med. Chem. 2002, 45,410 .

[17] Mourer, M.; Dibama, H. M.; Fontanay, S.; Grare, M.; Duval, R. E.; Finance, C.; Regnouf-de-Vains, J. B. Bioorg. Med. Chem. 2009, 17, 5496.

[18] Guo, M.; Zheng, C.J.; Song, M.X.; Wu, Y.; Sun, L.P.; Li, Y.J.; Liu, Y.; Piao, H.R. Bioorg. Med. Chem. Lett. 2013, 23, 4358. 